\title{
Asymptotic behaviors with convergence rates of distributions of negative-binomial sums
}

\author{
Tran Loc Hung ${ }^{1}$, Phan Tri Kien ${ }^{1}{ }^{*}$, Nguyen Tan Nhut ${ }^{2}$
}

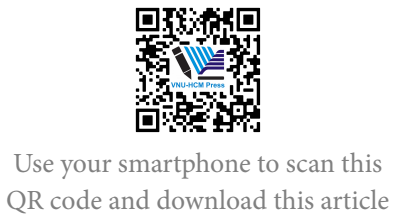

${ }^{l}$ University of Finance and Marketing, Vietnam

${ }^{2}$ Dong Thap Province, Vietnam

Correspondence

Phan Tri Kien, University of Finance and Marketing, Vietnam

Email: phankien@ufm.edu.vn

History

- Received: 2019-06-20

- Accepted: 2019-11-01

- Published: 2019-12-31

DOI :10.32508/stdj.v22i4.1689

\section{Check for updates}

\section{Copyright}

(c) VNU-HCM Press. This is an openaccess article distributed under the terms of the Creative Commons Attribution 4.0 International license.

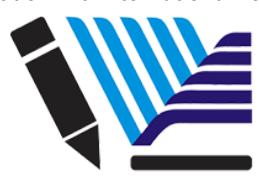

VNU-HCM Press

\begin{abstract}
The negative-binomial sum is an extension of a geometric sum. It has been arisen from the necessity to resolve practical problems in telecommunications, network analysis, stochastic finance and insurance mathematics, etc. Up to the present, the topics related to negative-binomial sums like asymptotic distributions and rates of convergence have been investigated by many mathematicians. However, in a lot of various situations, the results concerned the rates of convergence for negative-binomial sums are still restrictive. The main purpose of this paper is to establish some weak limit theorems for negative-binomial sums of independent, identically distributed (i.i.d.) random variables via Gnedenko's Transfer Theorem originated by Gnedenko and Fahim (1969). Using Zolotarev's probability metric, the rate of convergence in weak limit theorems for negativebinomial sum are established. The received results are the rates of convergence in weak limit theorem for partial sum of i.i.d random variables related to symmetric stable distribution (Theorem 1), and asymptotic distribution together with the convergence rates for negative-binomial sums of i.i.d. random variables concerning to symmetric Linnik laws and Generalized Linnik distribution (Theorem 2 and Theorem 3). Based on the results of this paper, the analogous results for geometric sums of i.i.d. random variables will be concluded as direct consequences. However, the article has just been solved for the case of $1<\alpha<2$, it is quite hard to estimate in the case of $\alpha \in(0,1)$ via the Zolotarev's probability metric.
\end{abstract}

Mathematics Subject Classification 2010: 60G50; 60F05; 60E07.

Key words: Negative-binomial sum, Gnedenko's Transfer Theorem, Zolotarev's probability metric, symmetric stable distribution, symmetric Linnik distribution, Generalized Linnik distribution

\section{INTRODUCTION}

We follow the notations used in ${ }^{1}$. A random variable $N_{r, p}$ is said to have negative-binomial distribution with two parameters $p \in(0,1)$ and $r \in N$, if its probability mass function is given in form

$$
\begin{aligned}
& \mathbb{P}\left(N_{r, p}=k\right)=\left(\begin{array}{c}
k-1 \\
r-1
\end{array}\right) p^{r}(1-p)^{k-1}, \\
& k=r, r+1, \ldots
\end{aligned}
$$

Let $\left\{X_{j}, j \geq 1\right\}$ be a sequence of independent, identically distributed (i.i.d.) random variables, independent of $N_{r, p}$. Then, the sum

$$
S_{N_{r, p}}=X_{1}+X_{2}+\cdots+X_{N_{r, p}}
$$

is called negative-binomial sum. It is easily seen that when $r=1$, the negative-binomial sum reduces to a geometric sum (see ${ }^{2,3}$ and $\left.{ }^{1}\right)$.

It is well-known that the topics related to negative-binomial sums have become the interesting research objects in probability theory. It has many applications in telecommunications, network analysis, stochastic finance and insurance mathematics, etc. Recently, problems concerning with negative-binomial sums have been investigated by Vellaisamy and Upadhye (2009), Yakumiv (2011), Sunklodas (2015), Sheeja and Kumar (2017), Giang and Hung (2018), Omair et al. (2018), Hung and Hau (2018), etc. (see ${ }^{1,4-9}$ ). In many situations, some problems on the negative-binomial sums have not been fully studied yet, therefore its applications are still restrictive.

Cite this article : Loc Hung T, Tri Kien P, Tan Nhut N. Asymptotic behaviors with convergence rates of distributions of negative-binomial sums. Sci. Tech. Dev. J.; 22(4):415-421. 
Therefore, the main aim of article is to establish weak limit theorems for normalized negative-binomial sums $\left(p_{n} / r\right)^{1 / \alpha} S_{N_{r, p}}$ via Gnedenko's Transfer Theorem (see ${ }^{10}$ for more details), where $1<\alpha<2, r \in N$, and $p_{n}=\theta / n$ for any $\theta \in(0,1)$. Moreover, using Zolotarev's probability metric, the rate of convergence in weak limit theorem for normalized negative-binomial sum $\left(p_{n} / r\right)^{1 / \alpha} S_{N_{r, p}}$ will be estimated. It is clear that corresponding results for normalized geometric sums of i.i.d. random variables will be concluded when $r=1$. From now on, the symbols $\underset{D}{\rightarrow}$ and $={ }^{D}$ denote the convergence in distribution and equality in distribution, respectively. The set of real numbers is denoted by $\mathbb{R}=(-\infty,+\infty)$ and we will denote by $\mathbb{R}=(1,2, \ldots\}$ the set of natural numbers.

\section{PRELIMINARIES}

We denote by $\mathfrak{X}$ the set of random variables defined on a probability space $(\Omega, \mathscr{A}, \mathbb{P})$ and denote by $C(\mathbb{R})$ the set of all real-valued, bounded, uniformly continuous functions defined on $\mathbb{R}$ with norm $\|f\|=\sup _{x \in \mathbb{R}}|f(x)|$. Moreover, for any $m \in \mathbb{N}, m<s \leq m+1$ and $\beta=s-m$, let us set

$$
C^{m}(\mathbb{R})=\left\{f \in C(\mathbb{R}): f^{(k)} \in C(\mathbb{R}), 1 \leq k \leq m\right\}
$$

and

$$
\mathscr{D}_{s}=\left\{f \in C^{m}(\mathbb{R}):\left|f^{(m)}(x)-f^{(m)}(y)\right| \leq|x-y|^{\beta}\right\}
$$

where $f^{(k)}$ is derivative function of order $k$ of $f$. Then, the Zolotarev's probability metric will be recalled as follows

Definition 1. $\left({ }^{11-13}\right)$. Let $X, Y \in \mathfrak{X}$. Zolotarev's probability metric on $\mathfrak{X}$ between two random variables $X$ and $Y$, is defined by

$$
d_{S}(X, Y)=\sup _{f \in \mathscr{D}_{S}}|\mathbb{E}[f(X)-f(Y)]| .
$$

Let $m=1$ and $s=2$, Zolotarev's probability metric of order 2 is defined by

$$
d_{2}(X, Y)=\sup _{f \in \mathscr{D}_{2}}|\mathbb{E}[f(X)-f(Y)]|,
$$

where $X, Y \in \mathfrak{X}$ and

$$
\mathscr{D}_{2}=\left\{f \in C^{1}(\mathbb{R}):\left|f^{\prime}(x)-f^{\prime}(y)\right| \leq|x-y|\right\}
$$

We shall use following properties of Zolotarev's probability metric in the next sections (see $\left.{ }^{11-13}\right)$. 1. Zolotarev's probability metric is $d_{s}$ an ideal metric of order $s$, i.e., for any $c \neq 0$, we have

$$
d_{s}(c X, c Y)=|c|^{s} d_{s}(X, Y),
$$

and with $Z$ is independent of $X$ and $Y$, we get

$$
d_{S}(X+Z, Y+Z) \leq d_{s}(X, Y) \text {. }
$$

2. If $d_{s}\left(X_{n}, X_{0}\right) \longrightarrow 0$ as $n \longrightarrow \infty$, then $X_{n} \stackrel{D}{\rightarrow} X_{0}$ as $n \longrightarrow \infty$.

3. Let $\left\{X_{j}, j \geq 1\right\}$ and $\left\{Y_{j}, j \geq 1\right\}$ be two independent sequences of i.i.d. random variables (in each sequence). Then, for all $n \in \mathbb{N}$,

$$
d_{s}\left(\sum_{j=1}^{n} X_{j}, \sum_{j=1}^{n} Y_{j}\right) \leq n . d_{s}\left(X_{1}, Y_{1}\right)
$$

The following lemma states the most important property of Zolotarev's probability metric which will be used in proofs of our results.

Lemma 1. Let $X, Y \in \mathfrak{X}$ with $\mathbb{E}|X|<\infty$ and $\mathbb{E}|Y|<\infty$. Then

$$
d_{2}(X, Y) \leq \sup _{f \in \mathscr{D}_{2}}\left\|f^{\prime}\right\| \cdot(\mathbb{E}|X|+\mathbb{E}|Y|)
$$


where $\left\|f^{\prime}\right\|=\sup _{w \in \mathbb{R}}\left|f^{\prime}(w)\right|$.

Proof. For any $x, y \in \mathbb{R}$ and $f \in \mathscr{D}_{2}$, by Mean Value Theorem we have

$$
f(x)-f(y)=(x-y) f^{\prime}(z)
$$

where $z$ is between $x$ and $y$. Moreover, since $f \in \mathscr{D}_{2}$, one has

$$
\left|f^{\prime}(z)\right| \leq \sup _{w \in \mathbb{R}}\left|f^{\prime}(w)\right|=\left\|f^{\prime}\right\| .
$$

Hence, we obtain following inequality

$$
f(x)-f(y) \leq|x-y| \cdot\left|f^{\prime}(z)\right| \leq\left\|f^{\prime}\right\| \cdot|x-y| .
$$

Therefore, for all $X, Y \in \mathfrak{X}$, we get

$$
\mathrm{d}_{2}(\mathrm{X}, \mathrm{Y})=\sup _{\mathrm{f} \in \mathscr{D}_{2}}|\mathbb{E}[\mathrm{f}(\mathrm{X})-\mathrm{f}(\mathrm{Y})]| \leq \sup _{\mathrm{f} \in \mathscr{D}_{2}}\left\|\mathrm{f}^{\prime}\right\| \cdot(\mathbb{E}|\mathrm{X}|+\mathbb{E}|\mathrm{Y}|)
$$

The proof is straight-forward.

In the sequel, we shall recall several well-known distributions which are related to limit distributions of non-randomly sums and negative-binomial sums of i.i.d. random variables.

We follow the notations used in $\left({ }^{1}\right.$, page 204). A random variable $Y$ is said to have symmetric stable distribution with two parameters $\alpha \in(0,2]$ and $\sigma>0$, denoted by $Y \sim \operatorname{Stable}(\alpha, \sigma)$, if its characteristic function is given in form

$$
\varphi_{Y}(t)=\exp \left\{-\sigma^{\alpha}|t|^{\alpha}\right\}, t \in \mathbb{R}
$$

A random variable $\xi$ is said to have symmetric Linnik distribution with two parameters $\alpha \in(0,2]$ and $\sigma>0$ denoted by $\xi \sim \operatorname{Linnik}(\alpha, \sigma)$, if its characteristic function is given by (see ${ }^{1}$, page 199)

$$
\varphi_{\xi}(t)=\frac{1}{1+\sigma^{\alpha}|t|^{\alpha}}, t \in \mathbb{R}
$$

A random variable $\Lambda$ is said to have Generalized Linnik distribution with three parameters $\alpha \in(0,2]$, and $r \in N$, denoted by $\Lambda \sim \operatorname{GLinnik}(\alpha, \sigma, r)$, if its characteristic function is given as (see ${ }^{1}$, page 216)

$$
\varphi_{\Lambda}(t)=\left(\frac{1}{1+\sigma^{\alpha}|t|^{\alpha}}\right)^{r}, t \in \mathbb{R} .
$$

\section{MAIN RESULTS}

From now on, let $r \in \mathbb{N}$ be a fixed natural number, $p_{n}=\frac{\theta}{n}$ for any, and $n \geq 1$. We first prove the following theorem.

Theorem 1. Let $\left(X_{j}, j \geq 1\right\}$ be a sequence of i.i.d. random variables with $\mathbb{E}\left|X_{1}\right|<\infty$. Assume that $\mathscr{S} \sim \mathscr{S}$ table $\left(\alpha,(\theta / r)^{1 / \alpha}\right)$, and $Y \sim$ Stable $(\alpha, 1)$ with $1<\alpha<2$. Then,

$$
d_{2}\left(\left(p_{n} / r\right)^{1 / \alpha} \sum_{j=1}^{n} X_{j}, \mathscr{S}\right) \leq n^{\frac{\alpha-2}{\alpha}}(\theta / r)^{\frac{2}{\alpha}} \cdot \sup _{f \in D_{2}}\left\|f^{\prime}\right\| .\left(\mathbb{E}\left|X_{1}\right|+\mathbb{E}|Y|\right)
$$

Proof. Let $\left(Y_{j}, j \geq 1\right\}$ be a sequence of i.i.d. copies of $Y$. Then, it is clear that

$$
\mathscr{S}={ }^{D}\left(p_{n} / r\right)^{1 / \alpha} \sum_{j=1}^{n} Y_{j} .
$$

Based on the ideality of order $s=2$ of Zolotarev's probability metric and according to Lemma 1, it follows that 


$$
\begin{aligned}
& d_{2}\left(\left(p_{n} / r\right)^{1 / \alpha} \sum_{j=1}^{n} X_{j}, \mathscr{S}\right)=d_{2}\left(\left(p_{n} / r\right)^{1 / \alpha} \sum_{j=1}^{n} X_{j},\left(p_{n} / r\right)^{1 / \alpha} \sum_{j=1}^{n} Y_{j}\right) \\
& =\left(p_{n} / r\right)^{2 / \alpha} d_{2}\left(\sum_{j=1}^{n} X_{j}, \sum_{j=1}^{n} Y_{j}\right) \\
& \leq\left(p_{n} / r\right)^{2 / \alpha} \cdot n \cdot d_{2}\left(X_{1}, Y_{1}\right) \\
& \leq n^{\frac{\alpha-2}{\alpha}}(\theta / r)^{2 / \alpha} \cdot \sup _{\mathrm{f} \in \mathscr{D}_{2}}\left\|\mathrm{f}^{\prime}\right\| \cdot\left(\mathbb{E}\left|\mathrm{X}_{1}\right|+\mathbb{E}|Y|\right)
\end{aligned}
$$

The proof is straightforward.

Remark 1 . Since $Y \sim$ Stable $(\alpha, 1)$, according to ( ${ }^{14}$, Corollary 5, page 305) then $\mathbb{E}|Y|<\infty$. Moreover, based on the finiteness of $\mathbb{E}\left|X_{1}\right|$ and $\left\|\mathrm{f}^{\prime}\right\|$, a weak limit theorem for normalized non-random sum will be stated from Theorem 1 as follows

$$
\left(p_{n} / r\right)^{1 / \alpha} \sum_{j=1}^{n} X_{j} \stackrel{D}{\rightarrow} \mathscr{S} \sim \text { Stable }\left(\alpha,(\theta / r)^{1 / \alpha}\right) \text { as } n \rightarrow \infty
$$

Proposition 1. Let $N_{r, p_{n}} N B\left(r, p_{n}\right)$. Then,

$$
\frac{N_{r, p_{n}}}{n} \rightarrow G \sim \operatorname{Gamma}(\theta, r), \quad \text { as } n \rightarrow \infty .
$$

Proof. Since $N_{r, p_{n}} \sim N B\left(r, p_{n}\right)$, the characteristic function of $N_{r, p_{n}}$ is given by

$$
\varphi_{N_{r, p_{n}}}(t)=\left(\frac{p_{n} e^{i t}}{1-\left(1-p_{n}\right) e^{i t}}\right)^{r}, \quad t \in \mathbb{R} .
$$

Hence, the characteristic function of $\frac{N_{r, p}}{n}$ is defined by

$$
\begin{aligned}
& \varphi_{\frac{N_{r}, p_{n}}{n}}(t)=\varphi_{N_{r, p_{n}}}(t / n)=\left(\frac{1}{1+\left(e^{-i t / n}-1\right) \frac{1}{p_{n}}}\right)^{r} \\
& =\left(\frac{1}{1+\left(\frac{e^{-i t / n}-1}{-i t / n}\right) \cdot \frac{i t}{\theta}}\right)^{r} .
\end{aligned}
$$

Letting $n \rightarrow \infty$, we conclude that

$$
\begin{aligned}
& \lim _{n \rightarrow \infty} \varphi_{\frac{N_{r, p}, p_{n}}{n}}(t)=\lim _{n \rightarrow \infty}\left(\frac{1}{1+\left(\frac{e^{-i t / n}-1}{-i t / n}\right) \cdot \frac{i t}{\theta}}\right)^{r} \\
& =\left(\frac{\theta}{\theta-i t}\right)^{r}=\varphi_{G}(t) .
\end{aligned}
$$

This finishes the proof.

Using Gnedenko's Transfer Theorem $\left(\mathrm{see}^{10}\right)$, a weak limit theorem for negative-binomial sum of i.i.d. random variables will be established as follows

Theorem 2. Let $\left\{X_{j}, j \geq 1\right\}$ be a sequence of i.i.d. random variables with $\mathbb{E}\left(\left|X_{1}\right|\right)<\infty$. Let $N_{r, p_{n}} \sim N B\left(r, p_{n}\right)$, independent of $X_{j}$ for all $j \geq 1$. Then,

$$
\left(p_{n} / r\right)^{1 / \alpha} \sum_{j=1}^{N_{r, p}} X_{j} \stackrel{D}{\rightarrow} \Lambda \text { as } n \rightarrow \infty,
$$

where $\Lambda \sim \operatorname{GLinnik}\left(\alpha, r^{-1 / \alpha}, r\right)$ with $1<\alpha<2$.

Proof. According to Proposition 1, we have

$$
\frac{N_{r, p_{n}}}{n} \rightarrow G \sim \operatorname{Gamma}(\theta, r) \quad \text { as } \quad n \rightarrow \infty
$$


where $\theta \in(0,1)$ and the density function of Gamma random variable $G$ is defined by

$$
f_{G}(x)=\left\{\begin{array}{ccc}
\frac{\theta^{r}}{\Gamma(r)} x^{r-1} e^{-\theta x} & \text { if } & x>0 \\
0 & \text { if } & x \leq 0 .
\end{array}\right.
$$

Furthermore, by Theorem 1, one has

$$
\left(p_{n} / r\right)^{1 / \alpha} \sum_{j=1}^{n} X_{j} \stackrel{D}{\rightarrow} S \text { as } n \rightarrow \infty,
$$

where $\mathscr{S} \sim$ Stable $\left(\alpha,(\theta / r)^{1 / \alpha}\right)$ whose characteristic function is given by

$$
\varphi_{S}(t)=\exp \left\{-(\theta / r)|t|^{\alpha}\right\}, t \in \mathbb{R} .
$$

On account of Gnedenko's Transfer Theorem $\left(\operatorname{see}^{10}\right)$, it follows that

$$
\left(p_{n} / r\right)^{1 / \alpha} \sum_{j=1}^{N_{r, p}} X_{j} \stackrel{D}{\rightarrow} \Lambda \text { as } n \rightarrow \infty,
$$

where $\Lambda$ is a random variable whose characteristic function is defined by

$$
\begin{aligned}
\varphi_{\Lambda}(t) & =\int_{0}^{+\infty}\left[\varphi_{\mathscr{S}}(t)\right]^{w} \frac{\theta^{r}}{\Gamma(r)} w^{r-1} e^{-\theta w} d w \\
& =\frac{\theta^{r}}{\Gamma(r)} \int_{0}^{+\infty} e^{-\left[\theta-\ln \varphi_{\mathscr{S}}(t)\right] w} w^{r-1} d w .
\end{aligned}
$$

Let us set $y=\left[\theta-\ln \varphi_{S}(t)\right] w$, then

$$
\begin{aligned}
\varphi_{\Lambda}(t) & =\frac{\theta^{r}}{\Gamma(r)} \int_{0}^{+\infty} e^{-y} y^{r-1}\left(\frac{1}{\theta-\ln \varphi_{s}(t)}\right)^{r-1} \frac{d y}{\theta-\ln \varphi_{s}(t)} \\
& =\left(\frac{\theta}{\theta-\ln \varphi_{s}(t)}\right)^{r}=\left(\frac{\theta}{\theta+(\theta / r)|t|^{\alpha}}\right)^{r} \\
& =\left(\frac{1}{1+(1 / r)|t|^{\alpha}}\right)^{r}
\end{aligned}
$$

The proof is immediate.

Next, the rate of convergence in Theorem 2 will be estimated via Zolotarev's probability metric by the following theorem.

Theorem 3. Let $\left\{X_{j}, j \geq 1\right\}$ be a sequence of i.i.d. random variables with $\mathbb{E}\left(\left|X_{1}\right|\right)=\rho \in(0,+\infty)$. Assume that $N_{r, p_{n}} \sim N B\left(r, p_{n}\right)$, independent of $X_{j}$ for all $j \geq 1$. Then,

$$
d_{2}\left(\left(p_{n} / r\right)^{1 / \alpha} \sum_{j=1}^{N_{r p_{n}}} X_{j, \Lambda}\right) \leq n^{\frac{\alpha-2}{\alpha}} \cdot(\theta / r)^{\frac{2-\alpha}{\alpha}} \sup _{f \in \mathscr{D}_{2}}\left\|f^{\prime}\right\| \cdot\left(\rho+\frac{2}{\alpha \sin \frac{\pi}{\alpha}}\right)
$$

, where $\Lambda \sim \operatorname{GLinnik}\left(\alpha, r^{-1 / \alpha}, r\right)$ with $1<\alpha<2$, and $\left\|f^{\prime}\right\|=\sup _{w \in R}\left|f^{\prime}(w)\right|$.

Proof. Let $\left\{\xi_{j}, j \geq 1\right\}$ be a sequence of independent, symmetric Linnik distributed random variables with parameters $\alpha \in(1,2)$ and $\sigma=1$,independent of $N_{r, p_{n}}$. Then, the characteristic function of $\operatorname{sum} \sum_{j=1}^{N_{r, p_{n}}} \xi_{j}$ is defined by

$$
\varphi_{\sum_{j=1}^{N_{r}, p_{n}} \xi_{j}}(t)=\left(\frac{p_{n} \varphi_{\xi_{1}}(t)}{1-\left(1-p_{n}\right) \varphi_{\xi_{1}}(t)}\right)^{r}=\left(\frac{p_{n}}{p_{n}+|t|^{\alpha}}\right)^{r} .
$$

Hence, the characteristic function of sum $\left(p_{n} / r\right)^{1 / \alpha} \sum_{j=1}^{N_{r, p_{n}}} \xi_{j}$ will be defined as follows

$$
\varphi_{\sum_{j=1}^{N_{j}, p_{n}} \xi_{j}}\left[\left(p_{n} / r\right)^{1 / \alpha} t\right]=\left(\frac{p_{n}}{p_{n}+\left(\left.\left(p_{n} / r\right)^{1 / \alpha} t\right|^{\alpha}\right.}\right)^{r}=\left(\frac{1}{1+\frac{1}{r}|t|^{\alpha}}\right)^{r}=\varphi_{\Lambda}(t) .
$$


Thus,

$$
\Lambda={ }^{D}\left(p_{n} / r\right)^{1 / \alpha} \sum_{j=1}^{N_{r, p n}} \xi_{j}
$$

On account of ideality of Zolotarev's probability metric of order $s=2$ it follows that

$$
\begin{aligned}
& d_{2}\left(\left(p_{n} / r\right)^{1 / \alpha} \sum_{j=1}^{N_{r, p_{n}}} X_{j, \Lambda}\right) \\
& =d_{2}\left(\left(p_{n} / r\right)^{1 / \alpha} \sum_{j=1}^{N_{r, p_{n}}} X_{j},\left(p_{n} / r\right)^{1 / \alpha} \sum_{j=1}^{N_{r, p_{n}}} \xi_{j}\right) \\
& =\left(p_{n} / r\right)^{2 / \alpha} d_{2}\left(\sum_{j=1}^{N_{r, p}} X_{j}, \sum_{j=1}^{N_{r, p_{n}}} \xi_{j}\right)==\left(p_{n} / r\right)^{2 / \alpha} \sum_{q=1}^{\infty}\left\{\mathbb{P}\left(N_{r, p_{n}}=q\right) \mathrm{d} 2\left(\sum_{j=1}^{q} X_{j}, \sum_{j=1}^{q} \xi_{j}\right)\right\} \\
& \leq\left(p_{n} / r\right)^{2 / \alpha} \sum_{q=1}^{\infty}\left\{\mathbb{P}\left(N_{r, p_{n}}=q\right) q \cdot d_{2}\left(X_{1}, \xi_{1}\right)\right\} \\
& =\left(p_{n} / r\right)^{2 / \alpha} \mathbb{E}\left(N_{r, p_{n}}\right) \cdot d_{2}\left(X_{1}, \xi_{1}\right)
\end{aligned}
$$

Since $\xi_{1} \operatorname{Linnik}(\alpha, 1)$ with $1<\alpha<2$, by Proposition 4.3 .18 in $\left({ }^{1}\right.$, page 212$)$, we have

$$
\mathbb{E}\left(\left|\xi_{1}\right|\right)=\frac{2}{\alpha \sin \frac{\pi}{\alpha}}
$$

On account of Lemma 1, one has

$$
\begin{aligned}
d_{2}\left(X_{1}, \xi_{1}\right) & \leq \sup _{\mathrm{f} \in \mathscr{D}_{2}}\left\|\mathrm{f}^{\prime}\right\| \cdot\left(\mathbb{E}\left|\mathrm{X}_{1}\right|+\mathbb{E}\left|\xi_{1}\right|\right) \\
& =\sup _{\mathrm{f} \in \mathscr{D}_{2}}\left\|\mathrm{f}^{\prime}\right\| \cdot\left(\rho+\frac{2}{\alpha \sin \frac{\pi}{\alpha}}\right)
\end{aligned}
$$

Therefore,

$$
\begin{aligned}
& d_{2}\left(\left(p_{n} / r\right)^{1 / \alpha} \sum_{j=1}^{N_{r, p_{n}}} X_{j, \Lambda}\right) \leq\left(p_{n} / r\right)^{\frac{2-\alpha}{\alpha}} \sup _{\mathrm{f} \in \mathscr{D}_{2}}\left\|\mathrm{f}^{\prime}\right\| \cdot\left(\rho+\frac{2}{\alpha \sin \frac{\pi}{\alpha}}\right) \\
& =n^{\frac{\alpha-2}{\alpha}} \cdot(\theta / r)^{\frac{2-\alpha}{\alpha}} \sup _{\mathrm{f} \in \mathscr{D}_{2}}\left\|\mathrm{f}^{\prime}\right\| \cdot\left(\rho+\frac{2}{\alpha \sin \frac{\pi}{\alpha}}\right)
\end{aligned}
$$

The proof is complete.

Remark 2. From Theorem 1, a weak limit theorem for normalized negative-binomial random sum will be stated as follows

$$
\left(p_{n} / r\right)^{1 / \alpha} \sum_{j=1}^{N_{r, p}} X_{j} \stackrel{D}{\rightarrow} \Lambda \sim \operatorname{GLinnik}\left(\alpha, r^{-1 / \alpha}, r\right) \quad \text { as } \quad n \rightarrow \infty
$$

\section{DISCUSSIONS}

In some situations, it is quite hard to establish the limiting distributions for negative-binomial sums of i.i.d. random variables. Meanwhile, if the limiting distribution of the partial sum is stated, the limiting distribution of corresponding negative-binomial sum will be established by the Gnedenko's Transfer Theorem (see ${ }^{10}$ ). Thus, in this paper, the asymptotic behaviors of normalized negative-binomial random sums of i.i.d. random variables have been established via Gnedenko's Transfer Theorem (Theorem 2).

Moreover, the mathematical tools have been used in study of convergence rates in limit theorems of probability theory including method of characteristic functions, method of linear operators, method of probability metrics and Stein's method, etc. Especially, the method of probability metrics is more effective. 
Using the Zolotarev's probability metric, the rates of convergence in weak limit theorem for partial sum and negative-binomial sum of i.i.d. random variables are estimated (Theorem 1 and Theorem 2).

It is worth pointing out that the Zolotarev's probability metric used in this paper is an ideal metric, so it is easy to estimate approximations concerning random sums of random variables. Furthermore, this metric can be compared with well-known metrics like Kolmogorov metric, total variation metric, Lévy-Prokhorov metric and probability metric based on Trotter operator, etc.

\section{CONCLUSIONS}

A negative-binomial distributed random variable with two parameters $r \in \mathbb{N}$ and $p_{n} \in(0,1)$ will reduce a geometric distributed random variable with parameter $p_{n} \in(0,1)$ when $r=1$. Hence, the analogous results for geometric sums of i.i.d. random variables will be concluded as direct consequences from this paper. However, the article has just been solved for the case of $1<\alpha<2$, it is very difficult to estimate in the case of $\alpha \in(0,1)$ via the Zolotarev's probability metric, but it will be considered in near future. Analogously, we can estimate the rates of convergence for negative-binomial sums of i.i.d. random variables for cases of $\alpha=1$ and $\alpha=2$.

\section{COMPETING INTERESTS}

The authors declare that they have no competing interests.

\section{AUTHORS' CONTRIBUTIONS}

All authors contributed equally and significantly to this work. All authors drafted the manuscript, read and approved the final version of the manuscript.

\section{ACKNOWLEDGMENTS}

The authors wish to express gratitude to mathematicians for sending their value published articles.

\section{REFERENCES}

1. Kotz S, Kozubowski TJ, Podgorsky K. Springer Science + Business Media, LLC; 2001.

2. Hung TL. On the rate of convergence in limit theorems for geometric sums. Southeast-Asian J of Sciences. 2013;2(2):117-130.

3. Kien TL, PT. The necessary and sufficient conditions for a probability distribution belongs to the domain of geometric attraction of standard Laplace distribution. Science \& Technology Development Journal. 2019;22(1):143-146.

4. Sunklodas JK. On the normal approximation of a negative binomial random sum. Lithuanian Mathematical Journal. 2015;55(1):150-158.

5. Vellaisamy P, Upadhye NS. Compound Negative binomial approximations for sums of random variables. Probability and Mathematical Statistics. 2009;29(2):205-226.

6. Sheeja SS, Kumar S. Negative binomial sum of random variables and modeling financial data. International Journal of Statistics and Applied Mathematics. 2017;2:44-51.

7. Omair M, Almuhayfith F, Alzaid A. A bivariate model based on compound negative binomial distribution. Rev Colombiana Estadist. 2018;41(1):87-108.

8. Giang LT, Hung TL. An extension of random summations of independent and identically distributed random variables. Commun Korean Math Soc. 2018;33(2):605-618.

9. Hung TL, Hau TN. On the accuracy of approximation of the distribution of negative-binomial random sums by the Gamma distribution. Kybernetika. 2018;54(5):921-936.

10. Gnedenko BV, Fahim G. On a transfer theorem. Dokl Akad Nauk SSSR. 1969;187(1):15-17.

11. Zolotarev VM. Approximation of the distribution of sums of independent variables with values in infinite-dimensional spaces. Teor Veroyatnost i Primenen. 1976;21(4):741-758.

12. Zolotarev VM. Probability metrics. Teor Veroyatnost i Primenen. 1983;28(2):264-287.

13. Zolotarev VM. Metric distances in spaces of random variables and their distributions. Mat Sb (NS), Volume 101(143). 1976;3(11):416-454.

14. Korolev V, Yu. Product representations for random variables with Weibull distribution and their applications. Journal of Mathematical Sciences. 2016;218(3):29-313. 